hollow of the diaphragm as soon as the abdomen was opened.

There are other conditions which one would think might naturally contribute to the development of lung diseases after abdominal operations. The operations are occasionally long and tedious, the patient leaving the table sometimes with a weak heart action, and the surface of the body more or less cold, and with blood deteriorated.

Any mucus lying in the bronhcial tubes is with difficulty expectorated for hours afterwards, and these conditions with a weak heart action are certainly factors to be avoided as far as possible. Then, again, the abdominal muscles play an important part in respiration and the post-operation abdominal soreness, and the pain caused by the divided muscles and suture line, whenever an attempt is made to cough or take a long breath, may readily favour the remaining in the bronchial tubes of more or less secretion. Patients the subject of emphysema, as will be seen from the table, seem more likely to suffer from bronchitis than from pneumonia.

The preventive measures which would seem to suggest themselves from a study of these cases relate in the first place to the anaesthetist. He should certainly be a welltrained man of judgement and experience, willing to give his whole attention to the administration of the anaesthetic, instead of watching the operator at his work. The greatest care should be observed in administering the ether slowly and sufficiently diluted not to exoite a hypersecretion of mucus in the air passages. Possibly a pre vious administration of scopolamine may prove to be a useful preventive measure. Stomach lavage in emergency cases is often useful by removing from the stomach food and liquid which may be vomited and then partly aspi rated. All parts of the body not necessarily exposed should be kept thoroughly wrapped in warm blankets.

There are undoubtedly predisposing causes of postoperative lung complications, among which may be mentioned age, sex, alcoholism, cachexia, and sepsis. Just in what way these different causes favour the development of lung complications is not easy in all cases to determine. Probably there are more influences than one, but it would seem from study of this group of cases that one important factor is the predisposition to inspiration of irritating and septic maiter from the mouth and pharynx. If the infection comes from the lymphatics and blood vessels it is not clear and is not evident in the autopsy room why there is a predilection for the right side and the base. In fact, I am so much impressed with the importance of preventing inspiration of mucus and vomitus from the pharynx that I have made it a standing rule in my wards that, beginning the evening of the day before the operation these patients shall have nothing but sterilized water to drink, and shall frequently use during that evening and the following morning an antiseptic mouth wash, and that the greatest possible care shall be taken by the anaesthetist during the period of anaesthesia and afterwards by the nurses in the ward to keep the mouth and pharynx clean and so far as possible free from an accumulation of mucus and secretions. Another point which I believe to be of importance is that these patients, after being returned to the ward, shall be so placed that by no possibility can a current of cold air pass over their chest.

I wish here to thank Dr. Johnson for his careful and painstaking work and assistance in preparing this paper.

THE late Miss Eliza Barber, formerly of Iublin, left $£ 5,000$ to the Meath Hospital for a new ward, to be called the "Eliza Barber Ward". $£ 2,000$ each to the Mercers" Hospital and the City of Iublin Hospital ; $£ 200$ each to the Adelaide Hospital and the Mater Misericordiae Hospital, and $£ 100$ each to the Convalescent Home, the Incurable Hospital, and the Cripples' Home, Usher's Island.

The Hon. Charles Arthur Ellis, of 36, Piccadilly and Shottermill, Surrey, who died on March 30th, bequeathed $£ 1,000$ each to the Metropolitan Convalescent Institution, the All Saints Convalescent Hospital, Eastbourne, the Victoria Hospital for Children, Chelsea, the Cripples' Home and Industrial School, Marylebone, the Royal Hospital for Incurables, the British Home for Incurables Earlswood Asylum for Idiots, and the Surgical Aid Society.
POST-ANAESTHETIC ACETONURIA :

THE SIGNIFICANCE OF DELAYED CHLOROFORM POISONING AND THE ADVANTAGES OF ETHER OVER CHLOROFORN IN ACUTE INFECTIVE CONDITIONS.

By LEWIS BEESLY, F.R.C.S.EDIN., RESIDENT SURGEON TO THE ROYAL HOSPITAL FOR SICK CHILDIIAN,
EDINBURGH.

Mr attention having been drawn to certain articles on acetonuria, acid intoxication, and delayed chloroform poisoning, I thought some investigations might with advantage be carried out with the endeavour to ascertain the significance of the presence of acetone in the urine in surgical cases, and with the object of explaining some of the toxic symptoms following the administration of general anaesthetics. With this in view I have during the past nine months carried out a series of examinations of the urine of patients before and after operation. These observations were made on children from 4 to 12 years old operated on by Mr. Stiles and Mr. E. Scott Carmichael at the Royal Hospital for Sick Children, Edinburgh.

I do not propose to discuss the various theories which have been advanced as to the origin of acetone or diacetic acid; the most recent information on this subject will be found in the papers by Stiles and Macdonald, ${ }^{1}$ Brackett, Stone and Low, ${ }^{2}$ and Kelly. ${ }^{3}$ In this paper I wish to report a series of investigations on the presence and excretion of acetone which are associated with certain clinical phenomena, the significance of which does not appear to have been sufficiently appreciated. I hope to be able to show that some of the unpleasant after-effects and untoward results of the administration of chloroform may be avoided or combated. The results have all been obtained from a careful quantitative estimation of the acetone from specimens of the total twenty-four hours excretion of urine, except in those cases in which either immediate operation or a fatal termination prevented it in these cases specimens were obtained from as much of the urine as could be collected. Every specimen was examined in addition by two qualitative tests for acetone ; a search was also made for diacetic acid, sugar, albumen, and casts.

Acetone is present in the urine in many different conditions, and is much more common than is usually supposed. There is so little in healthy arine, that it cannot be detected by colour tests, but by a quantitative estimation a trace is generally found. I have found it in every case after operation by the colour tests, except in one fatal case (see Chart 14).

The clinical similarity of the symptoms described as occurring in so-called acid intoxication, acidosis, acetonuria, and delayed chloroform poisoning seems to point to a similar metabolic disturbance in the organism, one of the ultimate products of which is acetone, and this product occurs with the greatest regularity after the administration of a general anaesthetic. I think I shall be able to show that a certain degree of intoxication or poisoning occurs after every surgical operation in which a general anaesthetic is administered. What determines the extent of the poisoning has yet to be discovered; I shall show, however, that its frequency and degree may be minimized by a careful choice of the anaesthetic and by suitable prophylactic measures.

Besides the cases of delayed chloroform poisoning which prove fatal, we meet with every degree of intoxication down to those in which the symptoms are so slight as to be overlooked altogether or misinterpreted, and my observations go to support the correctness of the view expressed by Stiles and Macdonald that these symptoms have frequently been erroneously attributed to delayed shock or sepsis.

I propose dividing the cases I have examined into two groups :

1.-Apparently Healthy Children whose Urine brfore Anaesthesia contained no Acetone or only the Faintest 'I'race of it.

Cases illustrating this group are shown in Charts 1, 2, 3 , and 4. It will be seen that the acme of acetone excretion is reached within the first twenty-four hours after operation. In these cases, while there were no clinical manifestations of chloroform poisoning, the presence of acetone in the urine showed that an abnos mal katabolic process had been dotermined by the anaesthetic. The 
curve shows that the acetone is produced rapidly and excreted quickly. The elimination of the acetone may be retarded by constipation. Hence it is probable that a certain amount of acetone is excreted by the bowel, and that constipation throws an extra burden on the kidneys. If a smart purge be given there is at once an acceleration in the excretion of the acetone, as is shown by the more rapid descent of the curve (Chart 3 ). The curve of descent may also be modified by the occurrence of sepsis. In a case in which the acme of acetone excretion was reached the day after operation and the normal decline appeared on the second day, the onset of sepsis at once caused a rise in the acetone excretion which was slightly maintained while the suppuration was progressing. The duration of the acetonuria may be seen by reference to Charts $1,2,3$, and 4 ; it generally lasts three or four days, but may be considerably prolonged by the presence of sepsis.

So far I have only referred to chloroform; when ether was employed a different result was observed. Charts 2 and 4 show that, while the acme of excretion was higher after ether than after chloroform, the rapidity of excretion was greater after the former.

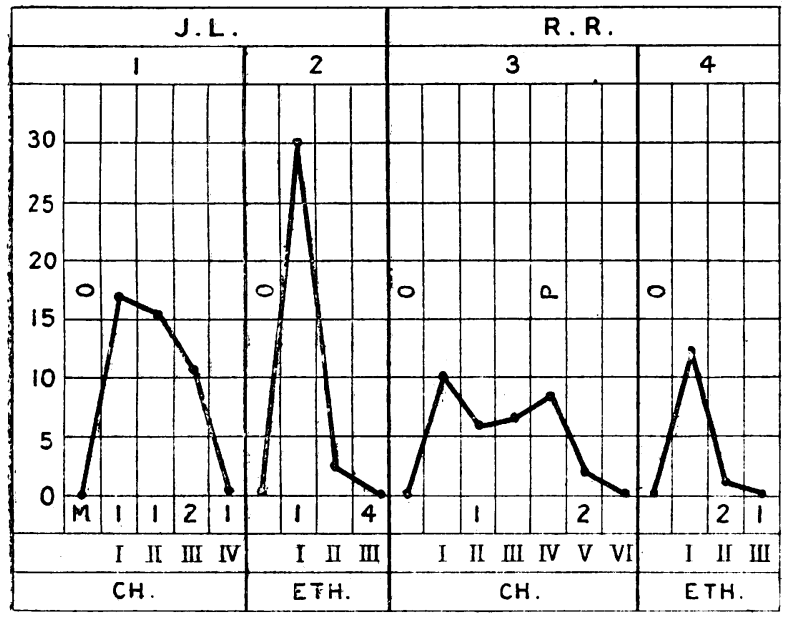

The numbers at the sides of the charts indicate in milligrammes the acetane in every 50 c.cm. of urine passed. The Roman gative.

No. 1.-J. L., 9 years. Osteotomy of femur. Chloroform 2 drachms. Anaesthesia of 15 minutes.

No. 2.-J. L. Osteotomy of the other femur. Ether 5 drachms. Anaesthesia of 11 minutes. Acme higher and descent more rapid than in No. 1 .

No. 3.-R. R., 7 years. Osteotomy of femur. Chloroform 4 drachms. Anaesthesia of 25 minutes. Shows the rise of the curve due to constipation and the drop following the action of the purgative.

No. 4.-R. R. Cuneiform osteotomy of the tibia and wiring. Ether $2 \mathrm{oz}$. Anaesthesia of 43 minutes. Note that th amount of acetone is less here than in Chart 2, although more ether was administered, a fact which seems to point to idiosyncrasy. The acetone in the urine did not give rise to any symptams of poisoning.

2.-Children whose Urine before Anaesthesia contained an appreciable quantity of Acetone, and who usually sufferte

from some Constitutional Condition more or less severe.

The cases in this group are "divided into (1) chronic and (2) acute acetonuria.

\section{Chronic Acetonuria.}

In chronic acetonuria the production and ixcretion of acetone is constantly proceeding. The quantity of acetone passed varies within wide limits. In some cases only $1.5 \mathrm{mg}$. are passed with every $50 \mathrm{c.cm}$. of urine; in others as much as 20.0 or $25.0 \mathrm{mg}$. are passed with every $50 \mathrm{c.cm}$. The less the amount of acetone passed the nearer do these cases approach those belonging to the first group, and they react similarly to the anaesthetic. The action of the anaesthetic in producing acetone is less marked the greater the degree of pre-existent acetonuria. The degree of acetonuria is variable in the same case day by day, sometimes, however, remaining fairly constant, but as soon as the cause of the acid intoxication is removed the deseent of the acetone curve is rapid. I have not found that the duration of the operation or any par- ticular anaesthetic has a more detrimental action on these cases than on those included in the first group.

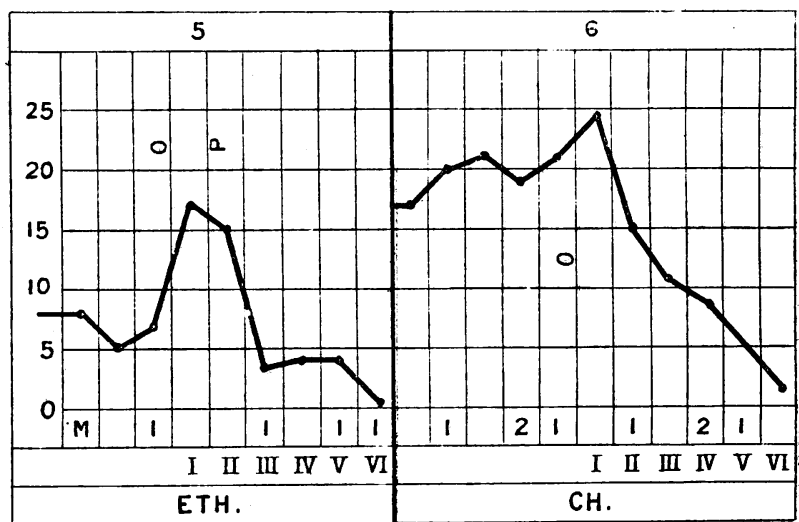

Chronic acetonuria. o, Operation ; $\mathrm{P}$, castor oil.

No. 5. Chronic Appendicular Abscess of Five Weeks' Note the fall of the curve after from a Daniell

No 6. Cishteen months duration Chloroform 5 drachms. Anaesthesia of 25 minutes. Suprapubic cystostomy. Neither of these patients showed any injurious effects after the anaesthetic.

Chronic acetonuria appears as the result of a constant absorption of toxins whose source may or may not be discoverable. It is of course obvious in such cases as are represented in Charts 5 and 6 but is more obscure in infantile paralysis, in which condition $I$ have found an average of $2 \mathrm{mg}$. to the $50 \mathrm{c.cm}$. of urine passed.

Brackett, Stone, and Low ${ }^{2}$ state that special care should be exercised in operating in cases of infantile paralrsis with extensive muscular wasting. Their reason appears to be that poisoning has more often occurred in this condition among their cases than in any other condition. I have not, however, seen any indication that poisoning is more frequent in such conditions. Daring the last year and a half twenty-five operations have been performed at the Royal Hospital for Sick Children on patients suffering from some deformity or weakness due to infantile paralysis.

The operations have included arthrodesis of the fnee and ankle and tendon transplantation. There were no deaths, and no case exhibited symptoms of acid intoriedtion. In three cases there was very slight romitiug on the evening of the operation. Chloroform, or chloroform and 12 per cent. alcohol, was the anaesthetic employed, except in 4 cases where ether was employed for comparison.

Delayed Acetone Excretion.

Before discussing acute acetonuria it will, I think, be advisable to enter into some account of the connexion which exists between the period of delayed acetone excretion and the clinical symptoms of poisoning which accompany this period. In the first group of cases I have shown that the normal acme of excretion occurs within twenty-four hours of operation. Cases are met with, however, where this normal elimination is altered. Some children, for no apparent reason, are more susceptible than others to the actions of anaesthetics, the result being a delayed acetone excretion, the acme not being attained for one, two, or three days after operation. Concurrently with this delayed excretion there occur toxic symptoms, which pass away as soon as the acme of excretion is reached.

The following case is a typical example of delayed acetone excretion.

7. Tuberculous Cerical Adenitis.-A. R., $5 \frac{1}{2}$ years, admittcd October 18th, 1905 .

History.-Always a healthy boy. About eleven montbs ago a swelling appeared in the neck; steady increase ever since two months ago a sudden enlargement was noticed ; never any pain. $\Lambda$ sister had a similar condition of the neck, and an pain. Aied of phthisis.

State on Admission. - A pale, ill-nourished boy, with a large cold abscess behind and below the angle of the jaw. Enlarged tonsillar and deep carotid glands, with periadenitis. Some isolated glands in the posterior triangle.

Operation.- October 19th. Ixcision of the abscess wall and all the glands by means of a large inverted J-shaped flar. 
Anaesthesia of 44 minutes, with mixture of chloroform and 12 per cent. absolute alcohol. Condition after Operation.-Some slight vomiting on

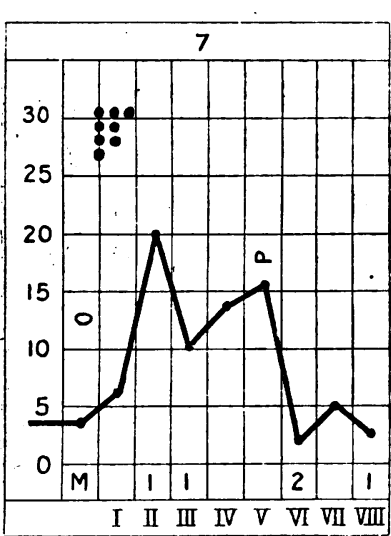

Delayed excretion. o, Operation

$P$, castor oil ; dots, vomiting. recovering consciousness. Slept quietly for some smell of acetone in the breath and repeated yomiting; restless, excited, and very thirsty. By 1 a.m., very thirsty. By 1 a.m., had become slightly coffeeground, pupils widely dilated; very restless, and calling out. Gradually be came sleepy, but wakened at intervals with vomiting no longer haemorrhagic. Odour of acetone very strong in the breath; this became less towards evening. The vomiting ceased, and a general improvement in the general improvement in the
boy's condition set in. The boy's condition set in. The remainder of the convalescence was uneventful. The acme of excretion was not reached until the second day after operation, when the symptoms of poisoning had passed off. The second rise in the chart points to constipation relieved by castor oil. Note the chart points to constipation relieved by

A graver form of delayed acetone excretion may occur after operation, and is shown by a sudden fall in the acetone curve instead of the gradual ascent. The symptoms accompanying this form resemble those of the former condition, but are more exaggerated. The prognosis in these cases is far more serious, and the two I have met with following chloroform narcosis ended fatally a few hours after operation. I shall refer to these cases later when describing acute acetonuria.

That less acetone is passed after operation than is expected suggests that either less is being produced or that some condition has arisen which prevents it being excreted. The first suggestion can, I think, be dismissed, for the odour of it in the breath affords undaubted proof of the presence of acetone.

That some condition has arisen which hinders or prevents the excretion of the acetone is shown on the one hand by the decrease of the acetone in the urine, and on the other by the appearance of the acetone in the breath associated with other toxic symptoms. Concurrently with the disappearance of the toxic symptoms and the odour from the breath there is an increase of acetone in the urine.

The frequency of this condition after chloroform as compared with ether at once suggests the possibility that there is some additional factnr present in chloroform narcosis which, besides producing acetonuria, acts on other organs, rendering them incapable of reacting to the strain of the acetone excretion. The examination of the liver and kidneys of animals experimentally anaesthetized, some with chloroform and some with ether, lias demonstrated that the action of chloroform is much more detrimental than ether.

These experimental results are fully corroborated by the practical evidence of the cases of acute appendicitis described below.

It is impossible to predict before operation whether any particular child will suffer from delayed excretion; experience shows that the condition is more frequent after certain operations than after others, but no definite rule can be Jaid down, except that if acute acetonuria (the condition next to be described) is present and chloroform be the anaesthetic emplozed, death will probably result.

There is no relation between delayed acetone excretion, duration of operation, or amount of anaesthetic administered, therefore I agree with the suggestion of Stiles and Macdonald that idiosyncrasy plays an important part in the reaction of the patient to chloroform. It is a well-recognized fact that certain individuals are more susceptible to poisons than others, and I consider that death following delayed chloroform poisoning is the result of the special reaction of the individual to the drug. Guthrie would have us believe that there is a particular pre-existent condition of the liver which is accountable for the post-anaesthetic poisoning of the patient, but if this be so the livers in all children suffering from delayed acetone excretion, which occurs as an antecedent stage in delayed chloroform poisoning, must be in a more or less fatty condition. I cannot understand his difficulty in accepting the theory of idiosyncrasy, especially when the pathological changes following the administration of chloroform have been so clearly shown by experimental work. Brackett, Stone, and $\mathrm{Low}^{2}$ state that

The comparatively small amount of acetone excreted in one of our cases shows that the quantitative estimation of the acetone excretion does not give a true index of the severity of the condition, and that the presence of any considerable amount of acetone in the urine should be considered as a probable sign of a more or less serious acid intoxication.

This is rather a sweeping statement to make on the strength of their single calculation. The experience of over 200 quantitative estimations has convinced me that a daily quantitative test of the acetone in the urine clearly indicates the course the case is taking. The important point is not so much the amount of acetone as whether the acme of excretion is or is not delayed. It is, I think, impossible to tell by any of the rough colour tests the difference between a urine containing one day $3 \mathrm{mg}$. of acetone per $50 \mathrm{c.cm}$., and the next day $6 \mathrm{mg}$. of acetone per 50 c.cm.; and on a difference as small as this symptoms of poisoning may depend. Misconceptions are likely to arise through an irregular and imperfect examination of the urine. In many of the cases described by previous observers no examination of the urine was made either before the operation or in the interval between the operation and the onset of the delayed poisoning which first drew attention to the presence of the acetone. Had the examination been made, two points of interest might have been disclosed: (1) That acute acetonuria was present before operation; or (2) that the anaesthetic at the operation had set up acute acetonuria combined with some degree of delayed excretion.

Acetonuria of varying degree follows the administration 
absolute alcohol. Anaestbesia of 40 minutes. The odour of acetone was perceptible the same evening, and was more marked throughout the next day. He suffered from extreme restlessness and thirst. The vomiting was most profuse but never reached the haemorrhagic stage. The symptoms passed off gradually on the third day after the operation, and had acetone excretion was not reached until the third day after operation.

No. 10. Tuberculous Cervical Adenitis.-A. P., $5 \frac{3}{4}$ years. Excision. Chloroform 6 drachms. Anaesthesia of 36 minutes. A pale, weakly boy, with a tendency to constipation. The glands had been present for nine montbs. Operation at midday; by 7 p.m. odour of acetone in the breath. Uncontrollable vomiting began one hour later; by 2 a.m. it had become slightly baemorrbagic; sod. bicarb. (30 gr.) were given fourhourly. He was extremely restless, excited, and cried out at times. The pupils were widely dilated. His thirst was excessive, and every few minutes be called for a drink. He became drowsy, and: vomited less during the following day, but that evening the persistent vomiting returned, though but that evening the persistent vomiting returned, though never becoming haemorrbagic. On the morning of the third of acetone, which had been present up to the night before had disappeared. He was much more comfortable, and could retain his milk. Convalescence after this was uninterrupted. The sod. bicarb. was rejuced in quantity, the urine having become alkaline. An enema was given, with slight result, on

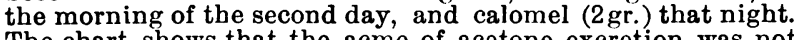
The chart shows that the acme of acetone excretion was not reached until the third day after the operation. Normal reached until the third day after the operation. Normal descent. In Charts 9 and 10 the acetone curve of the three
days following seems to show, when compared with the duradays following seems to show, when compared with the dura-
tion of the symptoms, that the kidneys were struggling to accommodate themselves to the amount of acetone requiring excretion. For the first two days they could not accomplish their work, with the result that symptoms of poisoning occurred. These symptoms of poisoning did not pass away until the kidneys had accommodated themselves to what was required of them. This was shown by the rise of the acetone curve to its acme on the third day. Just before or at the moment when the acme of excretion was reached the moment when the acme of excretion was reached the followed the course of a normal case, and there were no further symptoms of poisoning.

\section{Acute Acetonuria.}

Acute acetonuria is developed during the onset of acute infective conditions, and the quantity of acetone excreted is usually great. A large amount of acetone is formed. The liver and kidneys attempt to eliminate it, working at their highest pressure, and though unaccustomed to excreting acetone, they cope with the condition present, and no signs of poisoning appear. Should chloroform be administered at this stage, the extra amount of acetone formed from the action of the drug, combined with its special irritative qualities on the cells of the liver and kidneys, produces a sudden fall in the quantity of acetone excreted; this may in some cases amount to a total stoppage of elimination. Symptoms of poisoning ensue with great rapidity, and are more exaggerated than those described under delayed acetone excretion.

The difference in the action of chloroform in cases of chronic or acute acetonuria depends on whether or not the kidneys are accustomed to the excretion of acetone. The summation of detrimental influences, namely, the sudden acetonuria due to the infective condition, the extra acetonuria and pathological changes due to the action of the chloroform, cause a breakdown in the elimination.

That chloroform hinders the excretion of acetone to a greater extent than ether is seen by Charts $1,2,3$, and 4 and the following results substantiate this, showing that chloroform is a most dangerous anaesthetic in children suffering from acute acetonuria. Up to a year ago all the cases of acute appendicitis operated on at the Royal Hospital for Sick Children, Edinburgh, were anaesthetized with chloroform. The results show how disastrous this was, for death followed in a large proportion of the cases, no matter whether the infection was limited or general, or what the nature of the infection was proved to be by microscopic or cultural examinations. The following case is described in full, as it is a typical example of the condition I am attempting to describe.

\section{A CASE IN POINT.}

J. K., $8 \frac{1}{2}$ years. was in perfect health until October 20th 1905. On that evening she complained of some discomfor in the stomach, but slept well. The next day, October 21st, the pain was indefinite and much the same, not constant in character. She was kept in bcd. On October 22nd the pain became worse over the lower part of the abdomen, and now and then shot towards the right side. Sleep was much disturbed. On Octoher 23 rd the pain was rather less, but came on in spasms suddenly on the right side of the lower part of the abdomen. The bowels had acted daily, and there had been no vomiting or pain on micturition.

\section{State on Admission.}

Rather flushed. In no way distressed ; laughs and talks, and says her stomach is a "wee bit sair." Respiration easy and causing no pain even when deep. Tongue moist and clean. Abdomen moving freely and no swelling visible anywhere. No pain on light palpation over the abdomen, but some resistance on deeper pressure over the lower part on the right side. No mass to be felt and no dullness on percussion nothing to be felt by the rectum, which was not ballooned. Temperature $100.6^{\circ}$, pulse 114 ; leucocytes 28,000 ; glycogen reaction positive.

October 24th. Abdomen distinctly harder. Temperature $101.4^{\circ}$, pulse 124 .

\section{Operation.}

1 p.m. : Incision $2 \frac{1}{2}$ in. long in the right linea semilungris. Peritoneum opened. No adhesions or peritonitis visible. Caecum bound down by a mass at the brim of the true pelvis immediately below the ileo-caecal valve. General peritoneal cavity protected with gauze and the mass explored. Evil-smelling pus at once appeared, and was carefully swabbed away. It was thought advisable not to search for the appendix. $\dot{A}$ large drainage tube was passed down to the pelvis, and iodoform gauze packed around it to protect the rest of the abdomen. The incision was then partially closed, and the child sent back to bed apparently none the worse for the operation. The anaesthetic was chloroform and 12 per cent. absolute alcohol. Three drachms were used, and the
colde cent. absolute alcohol. Three drach
total anaesthesia lasted 18 minutes.

\section{Condition after Operation}

3 p.m.: There has been a little blood-stained vomit. and the pupils are widely dilated. She

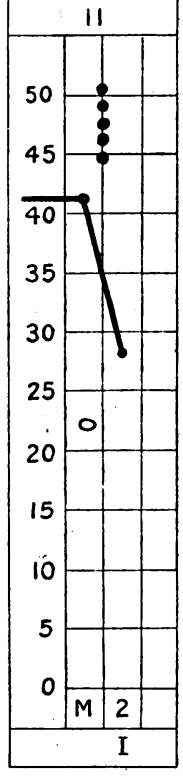

Acute

acetonuria. seems comfortable, and says she is in no pain. Pulse 108.4 p.m. : She is not looking so well, and has vomited a quantity of "coffee-ground" matter, and is constantly asking for drinks. Temperature $100^{\circ}$, pulse 118 . 5 p.m. : Has again vomited "coffee-ground" matter, and is getting restless, and inclined to throw ber arms about. Pulse not so strong. Saline and adrenalin given subcutaneously. Stomach washed out with saline and sodium bicarbonate, some of the latter left within. She is lying with her pupils widely dilated and ber eyes half shut. There is a strong smell of acetone in her breath. Stimulants given. 6 p.m. : Is more restless, and has some "air hunger"; pulse hardly so good. Will answer when spoken to. Has vomited everything left in stomach; blood-stained. 8 p.m.: Is much worse Calling out and screaming with inworse. Calling out and screaming, with inhunger" more pronounced. Strong smell of acetone. Very thirsty. Has not vomited since last note. 10 p.m.: Increasing difficulty in swallowing; harder to rouse; breathing laboured; strong smell of acetone; has not vomited. 11.30 p.m. : Has again vomited "coffee-ground" material; some twitching of the face and fingers; unconsciousness coming on; the respirations are much slower; she on; the respirations are much slower ; she with an effort takes a deep inspiration; no pulse to be felt. 12.20 : Death ; temperature $106^{\circ}$. Microscopic and cultural examinations of the pus showed a streptococcal infection.

Post-mortem examination: A gangrenous appendix fixed to the edge of the pelvis. The right ovary formed the right wall of the abscess, left wall formed by the pelvic colon. No concretion and no general peritonitis. The liver, heart, kidneys, and spleen showed very extensive fatty degeneration; there were some haemorrhages in the spleen.

Notice in the chart (No. 11) the sudden descent of the acetone curve denoting the diminished excretion of acetone the black dots represent the vomiting; the high degree of acetonuria before operation.

On looking back over the cases of acute appendicitis operated on at the Royal Hospital for Sick Children, Edinburgh, from January, 1904, the same symptoms may be traced after chloroform anaesthesia-the excitement, delirium, coma, the persistent uncontrollable vomiting becoming haemorrhagic or "coffee-ground," and ending generally in death. Sometimes the poisoning came on at once, sometimes it was delayed for a day or two.

As the deaths described as due to delayed chloroform poisoning so closely resembled the deaths occurring after operations with chloroform on children suffering from an acute infective condition combined with acetonuria Mr. Stiles suggested using ether as the anaesthetic instead 
Cases of Acute Perforating Appendicitis Operated on in the Royal Hospital for Sick Children, Edinourgh.

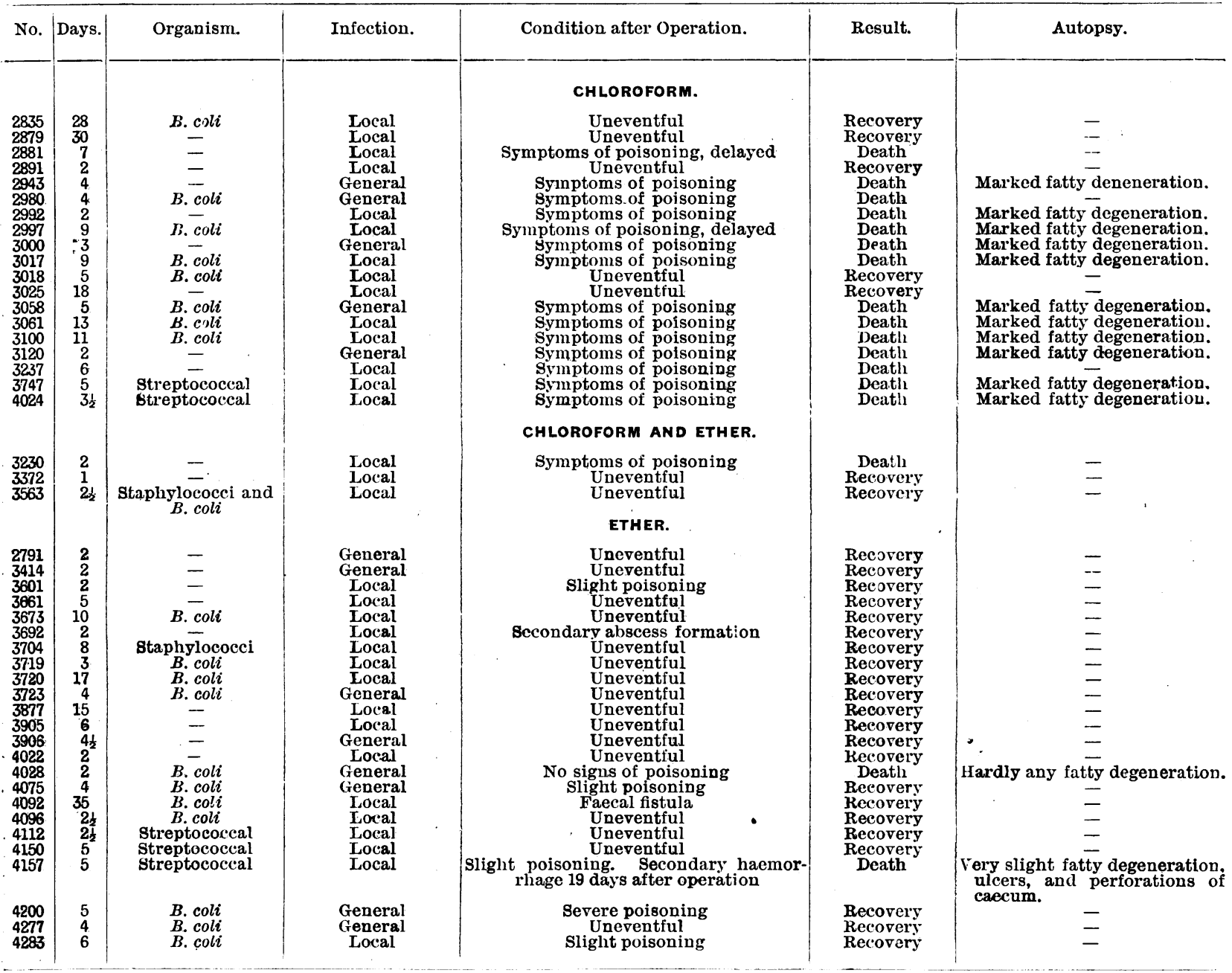

of chloroform. The results of this change prove beyond doubt which is the best anaesthetic to use in such cases. In the above table will be found a list of the cases treated in this manner. There is no reason to suppose that the cases this year were milder, or that the diagnosis was made earlier, and that the cases were sent in sooner to the hospital. The microscopic examination of the pus and the cultures from it show that just the same conditions were present as before.

The results of the foregoing statistics may be summarized as follows: Out of 19 cases of acute appendicitis operated on under chloroform, 14 died. All of these exhibited symptoms of acid intoxication or acetone poisoning ; 11 autopsies were granted, and in all of these well-marked fatty degeneration of the liver, kidmeys, and heart was present. Of the recoveries, 3 were operated on eighteen, twenty-seven, and thirty days after the onset of symptoms. It is probable that in these cases chronic and not acute acetonuria was present, and that the danger of the anaesthetic was thus lessened.

Out of 3 cases operated on under a mixture of chloroform 1 part and ether 2 parts, 1 died with symptoms of acetone poisoning. Out of 24 cases operated on under ether, only 2 died, and that not as the result of acid intoxication. In one case the necropsy showed hardly any fatty degeneration, in spite of general peritonitis having been present for four days. There were no symptoms of acetone poisoning in this case (Chart 13). The second death was from secondary haemorrhage nineteen days after the operation; 5 of the patients exhibited mild symptoms of acid intoxication, but only in 1 were they at all alarming. No. 4,092, with an appendicular abscess of thirty-five days' duration, suffered from chronic and not acute acetonuria.

These results show, as clearly as the experiments on animals, that the action of ether is far less harmful than chloroform. The acetone curve following ether adminis- tration in acute acetonuria is shown in Chart 12 . The curve shows the acute acetonuria, the rise after the anaesthetic, and the gradual decline, more prolonged than in normal ether cases, because of the suppuration present.

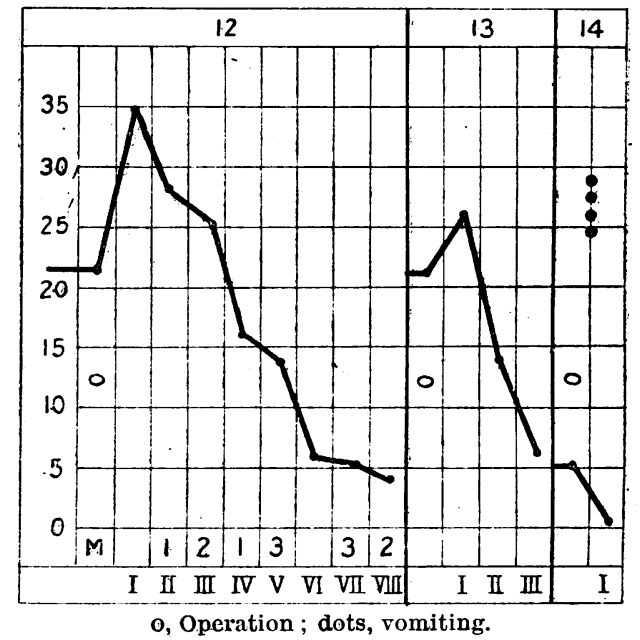

No. 12. Acute Perforating Appendicitis.-B. M., 5 years. Anaesthesia of 40 minutes with 6. oz. ether. A localized abscess and gangrenous appendix of three days' duration. Microscopic and cultural examinations ghowed streptococcal infection. Appendicectomy and drainage. An uneventful infection. Appendicectomy and drainage. An uneventful
recovery, with no symptoms of poisoning. Wound healed in recovery, with no symptoms of poisoning. Wound healed in
thirty-one days. There was no diacetic acid before or after the operation.

The next case is of particular interest, as although death occurred it was not due to an acid intoxication. 
No. 13. Acute Perforating Appendicitis and General Peritonitis-D. G., 113 vears. Two days' duration. Anaesthesia of 45 minutes with $6 \mathrm{oz}$. ether. Appendicectomy and multiple drainage. Microscopic and cultural examinations showed $B$, coli.

The boy recovered well from the operation, and remained very comfortable for forty-four hours. At the end of that time he became very cold, and the pulse-rate increased, but he appeared quite cheerful, and said he was free from pain. Stimulants, warm blankets, and hot bottles were applied, and shortly afterwards he fell asleep. About four hours later be breathing became difficult and his pulse imperceptible, and he died suddenly.

The chart shows the acute acetonuria before the operation, the rise due to the anaesthetic and the decline with the exthe rise due to the ana

There were never any symptoms of poisoning; no odour of acetone; no restlessness; no excitement; no coma. He vomited twice on the second day, once immediately after being given some hot milk and once just after a dose of castor oil. His progress was excellent as regards the excretion of urine and acetone. There was no diacetic acid before or after operation.

The following case is inserted for comparison with cases 12 and 13, and shows the disastrous result of chloroform in an acute infective condition, acute acetonuria being present.

No. 14. Acute Infective Osteomyelitis of Tibia.-A. M., a boy, aged 8 years, gave a history of a slight injury to his heel about eight days before.

On admission the leg was red, painful, and oedematous. There were large bullae scattered over the ankle and a sloughy sore over the heel. The part was hot, and fluctuation was detected in the lower third of the leg. The operation was performed under chloroform and 12 per cent. absolute alcohol. performed under chloroform and 12 per cent. absolute alcohol. Seven drachms were used, and the anaesthesia lasted for 52
minutes. The diaphysis of the tibia was removed subperiosteally, the bone having been first trephined to determine its condition.

The patient was rather collapsed after the operation but improved considerably after being subcutaneously infused with $15 \mathrm{oz}$. of saline and adrenalin, and thoroughly warmed and stimulated.

About three hours after the operation he became very noisy, screaming and tearing at his bandages. There was a strong smell of acetone in the breath, and the pupils became widely dilated; soon after, without the slightest effort, he brought up a large quantity of "coffee-ground" material. The restlessness became more marked, and it became impossible to leave him for a moment. The haemorrhagic vomiting continued, and he complained of great thirst, continually shouting for drink. The pulse was 150 to 160 and small, but fairly regular. Nine hours after the operation he began to strugrle for and shortly afterwards became comatose, dying within eleven hours of the operation.

The chart shows the sudden fall in the acetone excretion after the operation. The case also shows that the acnte acetonuria need not necessarily be of great degree to aid the chloroform in producing symptoms of acetone poisoning

Kelly $^{3}$ has shown the frequency of acetone in acute appendicitis before operation, and his account of the convalescence of the patients agrees with my own observations, though had he estimated the acetone by the quantitative method his results would probably have tallied still more closely. Ether was the anaesthetic used throughout in the cases he reports, and of these only two died-a case of acute appendicitis with the symptoms usually associated with chloroform poisoning, and a case of acute osteomyelitis after repeated operations. Brackett Stone, and Low ${ }^{2}$ give a list of conditions in which acetone has been found, but fail-and this I think important-to discriminate between acetonuria of sudden occurrence and of long standing. More information regarding its presence in other conditions is needed before its true significance can be fully determined, and it would be of advantage to the surgeon particularly to know whether or not he was dealing with a case of acute acetonuria.

\section{Treatment.}

Acetonuria should be treated by accelerating and stimulating the excretions, and by the administration of sodium bicarbonate pushed in such doses that the urine becomes alkaline. Morse, ${ }^{4}$ in his paper on the acid intoxication of infancy, states that attacks of indigestion can often be aborted by giving sodium bicarbonate. This suggested to me that the onset of chloroform poisoning might perhaps be prevented or mitigated by giving that drug beforehand. Two cases of extensive tuberculous cervical adenitis were selected, and placed on sodium bicarbonate (gr. 15 t.i.d.) for eight days before the operation. The results were excel- lent, the patients vomited very slightly on recovering consciousness, but at no time did they exhibit any symptoms of poisoning. They took and retained food the same evening, and merely suffered inconvenience from the pain attending the wound. The ward sister said that they appeared to be hardly affected at all, and did not require the constant attention usually called for by gland cases. All children admitted to the Royal Hospital for Sick Children, Edinburgh, suffering from glands in the neck are now placed on this treatment before operation, and the results justify the procedure.

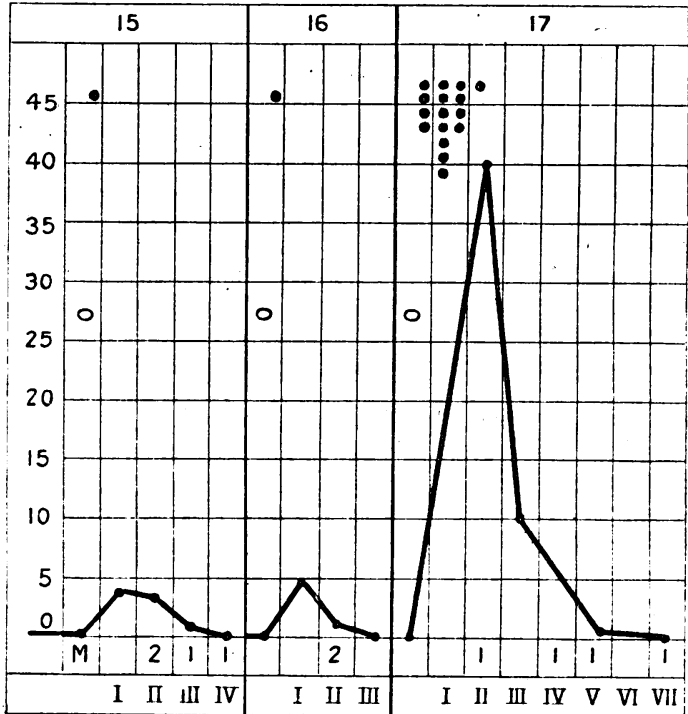

o, Operation ; dots, vomiting

Charts 15, 16, 17 indicate the difference between two cases of glands with preparatory treatment and a case without that treatment. In the former it will be seen that there was either very little metabolic disturbance, or that the results of the metabolism were rendered harmless by the presence of the alkali. The curve shows the quantity of acetone reduced, and that the acme occurred within the first twenty-four hours.

No. 15. Tuberculous Cervical Adenitis.-G. McO., $6 \frac{3}{4}$ years. Excision; anaesthesia of 47 minutes with chloroform (7 drachms). Preparatory course of treatment with sodium bicarbonate. No symptoms of poisoning.

No. 16. Tuberculous Cervical Adenitis. - R. W. 6 years. No. 16. Tuberculous Cervical Adenitis. $-\mathrm{R}$. W. 6 years. (3 drachms). Preparatory course of treatment with sodium (3 drachms). Preparatory course of treatment with sodium
bicarbonate for 8 days before operation. No symptoms of poisoning.

No. 17.'Tuberculous Cervical Adenitis.-K. M., 6- years. Excision ; anaesthesia of 62 minutes under a mixture of chloroform and ether. The chart shows the uncontrollable vomiting which during the morning after the operation became haemorrhagic and the delayed acme of exeretion. There was a marked odour of a marked odour of acetone in the breath, and the pupils wer These symptoms passed off the second day after operation, These symptoms passed off the seco

The treatment of cases showing symptoms of poisoning must be directed to removal of the acid intoxication, by stimulating the excretions. In the milder cases it is sufficient to administer sodium bicarbonate. Should there be any suspicion of serious acid intoxication every means must immediately be employed to counteract it; if there be hesitation or waiting, no treatment will prove of value. The patient's skin must be made to act freely either by vapour baths or blankets and hot bottles. Sodium bicarbonate must be introduced in various ways into the stomach after thorough lavage, by rectal injections, or subcutaneously in combination with saline infusions. At the first sign of collapse free stimulation of the patient must be commenced, and I agree with Kelly that subcutaneous saline infusions combined with adrenalin improve the pulse and general condition of the patient.

Although the average number of patients in the surgical wards of the Royal Hospital for Sick Children, Edinburgh, is about 730 a year, I have not during the last year seen a single case comparable to the series described by Brackett, Stone, and Low, ${ }^{2}$ of acid intoxication prior to operation or coming on during convalescence. Hubbard's ${ }^{5}$ 
statement as to acetone-that " its presence without symptoms has no effect on operative treatment or prognosis" - seems to me to require considerable qualification. I think I have shown conclusively that where acute acetonuria is present it requires very careful consideration, and that it renders the prognosis distinctly less favourable, especially when chloroform is the anaesthetic employed. If Hubbard refers merely to chronic acetonuria I agree with him.

Conclusions.

1. That two separate conditions should be recognizedacute and chronic acetonuria.

2. That ether and chloroform invariably induce a temporary acute acetonuria which may be very detrimental even to an apparently healthy organism.

3. That this acute anaesthetic acetonuria is accom panied by symptoms of acid intoxication, sometimes ending in death, when the kidneys are unable to cope with the increased formation of acetone by a corresponding capability of excretion.

4. That although ether may produce a greater acetonuria, this is less harmful than that produced by chloroform, because ether is less injurious to the cells of the liver and kidneys, and thus does not hinder their power of elimination.

5. That the more plentifully and rapidly excretion is carried on the less serious is the poisoning.

6. That the effects of the poisoning are mitigated by the administration of alkalies, which may also be given with advantage before operation if poisoning be anticipated.

7. That the usual risks of anaesthesia are not increased by pre-existent chronic acetonuria.

8. That anaesthesia is dangerous with pre-existent acute acetonuria, especially if the anaesthetic is chloroform.

9. That a guarded prognosis must always be given when acute acetonuria is present with symptoms of poisoning.

10. That death following the administration of chloroform with symptoms of poisoning may be due to the idiosyncrasy of the patient.

I am indebted to Dr. McAllum, Anaesthetist to the Hospital, for the anaesthetic notes, and my sincere thanks are due to the Sister of the Wards for her kindly co-operation, as without her assistance I could not have carried out a great part of the work.

\section{Test for the Quantitative Estimation of Acetone} in the Urine.

In examining urines, I have used the method described in Hoppe-Seyler's Physiological Chemistry; this takes on the average four hours to complete. The twenty-four hours' collection having been obtained $50 \mathrm{c} . \mathrm{cm}$. is taken, and if it is not acid a drop or two of a 50 per cent. solution of acetic acid is added. This is distilled very slowly, and generally occupie two hours. When the distillate amounts to $45 \mathrm{c.cm}$. it should be mixed with a little calcium carbonate and about 25 or $30 \mathrm{~cm}$ of cold water and then redistilled. This will ocoupy about half an about half an hour. The distillate is then acidified with little of a 1 to 7 solution of strong sulphuric acid and then redistilled. The final distillate is then ready for testing. A known amount of a decinormal iodine solution is run from a burette into the distillate, and a sufficient amount of nitric-free caustic potash is added to at once discharge the brown colour The mixture is then shaken for half a minute and then allowed to stand for five minutes. Strong hydrochloric acid is now added until the mixture becomes acid, when it should at once become brown again if there is a superfluous amount of iodine present. Finally, a decinormal solution of sodium thiosul. present. Fin in f a decinormal (odine is neutralized. This is best estimated by adding a starch solution before all the iodine is nentralized. The amount of thiosulphate used will correspond to the amount of superfluous iodine which bas not united with the acetone to form iodoform ; from this it is easy to estimate how much iodine has united with the acetone. It bas been chemically determined that 1 c.cm. of a decinormal iodine solution $=0.967 \mathrm{mg}$. of acetone.

ther tests for acetone employed

To a specimen of the urine add a drop or two of a freshly-prepared solution of sodium nitro-prussiate, two or three drops of caustic soda, and a little acetic acid. Shake, and if acetone is present the foam will be of a purple colour, the depth of the colour depending on the amount of acetone present.

To a specimen of the urine add a few drops of a freshlyprepared solution of sodium nitro-prussiate and some 10 per cent. caustic potash solution. A red colour is produced, which if left to stand becomes canary yellow, the red colour returns on adding a drop or two of acetic acid and shaking.

The object of this paper has been to show that certain symptoms after operation sometimes followed by death, which are either unaccounted for or attributed to wrong causes, are really due to an acid intoxication of sudden occurrence and variable intensity, originated by the anaesthetic and indicated by the degree of acetone excretion in the urine. Although other circumstances may sometimes have contributed to the fatal result, the intoxication is by itself capable of producing death.

1 Stiles and Macdonald, Delayed Chloroform Poisoning, Scottish Medical and Surgical Journal, August, 1904. " Brackett, stone, and Low, Acetonuria Associated with Death after Anaesthesia, Boston Medical and Surgical Journal, July, 1904. ${ }^{3} \mathrm{Kelly}$, Acid Intoxication, Annals of Surgery, February, 1905. 4 Morse. Acid Autointoxication in Infancy and Childhood, Archives of Pediatrics, August, 1905 and Surgical Journal, June, 1905.

\section{SUBPHRENIC ABSCESS COMPLICATING EM- \\ PYEMA : RESECTION OF RIBS : CURE.}

By EDWARD W. ARCHIBALD, M.D. ASSISTANT SURGEON, ROYAL VICTORIA HOSPITAL; ASSOCIATE SURGEON

IT was Maydl's well-known monograph in 1894, with its analysis of all the cases of subphrenic abscess published before that year, that stimulated scientific interest in this subject. The decade since 1894 has seen two further articles of a collective character, which have brought our knowledge of the question well up to date. Grueneisen reports upon 60 cases from Koerte's clinic alone; while Perutz ${ }^{2}$ gives a most excellent summary of 208 cases from the literature of the last ten years.

An examination of these sources reveals the clinical fact, possibly not thoroughly realized hitherto, that subphrenic abscess as a complication of an intrathoracic infection, is a decidedly rare event. Of the 447 cases reviewed in the three articles mentioned, only 21 are given as owing their origin to intrathoracic disease Of these 21 cases we may probably exclude 4 as lacking proof, 2 of Maydl's cases (Nos. 4 and 8 in Paget's review ${ }^{3}$ ) and 2 of Grueneisen's (Cases Lvi and Lvir loc. cit.). Adding to the 17 remaining the case here reported, we get a total of 18 cases. The analysis of this material reveals certain facts of interest. Thus, as to the primary lesion, we find that it was in 6 cases an empyema following pneumonia, in 7 empyema following an undetermined source of infection (influenza? pleurisy ?), in 1 an abscess of the lung, in 1 gangrenous bronchitis with pleurisy, in 2 gangrene of the lung, and finally, in 1 tuberculosis of the lung, the focus being in the right lower lobe. Although the cause of the empyema in our own case is set down as "probably grippe," the finding later of pneumococci in the pus renders the diagnosis of pneumonia a more likely one.

In the reports collected by Maydl, antedating 1894, all but one of which represented post-mortem findings, the diaphragm was found perforated; while in later cases, almost all treated by operation, no perforation could be discovered-from which we may deduce that the abscess under the diaphragm originates as a metastatic lesion, by way of the lymphatics joining one cavity with the other but that later, as the result of prolonged contact with pus, the diaphragm is gradually destroyed at one or more spots.

The mortality figures offer, as usual, a strong argument in favour of operation on modern lines. Of Maydl's 7 cases before 1894 only one came to operation. This patient recovered; the other six died. Of the 11 cases since 1894, 2 were accidental autopsy findings; while of the remaining 9 patients, who were all operated upon, 7 recovered, 1 dying as the result of a second undiscovered subphrenic abscess.

The difficulty of recognizing the subphrenic abscess before operation in cases in which it complicates a pleural effusion, and in which gas is not present, is well illustrated by the simple fact that, so far as I can ascertain, the condition has been uniformly missed. The abscess is discovered only during operation for a supposedly simple empyema. Frequently, as in the present case, the 